\title{
Beilinson conjecture for finite-dimensional associative algebras
}

\author{
D. Kaledin*
}

\section{Contents}

1 Beilinson conjecture.

1.1 R-Hodge structures. . . . . . . . . . . . . . . . . . . 3

1.2 Absolute Hodge cohomology. . . . . . . . . . . . . . . . . . 3

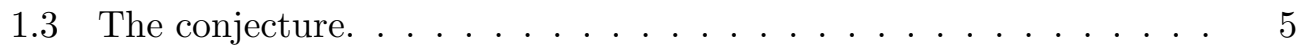

2 Kato's reformulation. 6

3 Non-commutative version 9

3.1 DG algebras. . . . . . . . . . . . . . . . . 9

3.2 The conjecture. . . . . . . . . . . . . . . . 11

3.3 Finite-dimensional algebras. . . . . . . . . . . . . 14

\section{Introduction}

The phrase "Beilinson conjectures", or, more precisely, "Beilinson's conjectures on special values of $L$-functions" refers to a set of conjectures formulated by A. Beilinson in [Be]. The subject is, roughly speaking, relations between algebraic $K$-groups of a smooth proper algebraic variety $X$ over $\mathbb{Q}$ and its de Rham cohomology groups equipped with the Hodge structure. The conjectures explain and tie together nicely several disparate known facts and present a unified picture of great beauty and great simplicity. One wants to believe in them to such an extent that there are some papers where the author states right away that everything takes place in a "Beilinson World" - the world where the conjectures are true. However,

*Partially supported by RFBR grant 12-01-33024, Russian Federation government grant, ag. 11.G34.31.0023, and the Dynasty Foundation award. 
the conjectures are notoriously difficult: in the 30 years that passed since $\mathrm{Be}$, we are not much closer to knowing whether we live in a Beilinson World or not.

Formally, the eventual goal of the conjectures is to predict, up to a rational multiple, the values of $L$-functions of the algebraic variety $X$ at integer points. This follows earlier conjectures by Deligne, where the values were predicted in some cases, and expressed in terms of the "period matrix" relating natural $\mathbb{Q}$ structures in the de Rham cohomology and the Betti cohomology of $X$. To get the individual values, one has to also take account of the Hodge filtration on de Rham cohomology; in the end, for every integer, one produces an $\mathbb{R}$-vector space with two independent $\mathbb{Q}$-structures, and the determinant of the corresponding transition matrix should give the $L$-values.

However, this only works in some cases; in the general case, one ends up with a $\mathbb{R}$-vector space with only one obvious $\mathbb{Q}$-structure, and it is not clear how to produce a number out of that. Beilinson's insight was to add algebraic $K$ groups to the picture. The vector space in question is the target of a certain natural "regulator map" from $K$-groups; Beilinson then conjectures that, roughly speaking, the regulator map becomes a isomorphism after tensoring with $\mathbb{R}$, so that we obtain a second $\mathbb{Q}$-structure, and that the determinant of the resulting period matrix gives the $L$-values.

In the present note, we are only concerned with the first part of the conjectures - namely, with the prediction that the regulator map becomes an isomorphism after tensoring with $\mathbb{R}$ (this is why we use the singular "conjecture" in the title). We only do two things: we observe that the conjecture can actually be stated so that it makes sense for a non-commutative algebraic variety, and then we observe that the conjecture thus stated is almost trivially true for finite dimensional associative algebras (we do not really give a complete proof, but we do give a detailed sketch). This is boring, but not as boring as the corresponding commutative statement finite-dimensional associative algebras are not as trivial as the commutative ones. In fact, it is the basic principle of non-commutative algebraic geomery that every variety is derived-affine, so that were we able to extend our observation from algebras to DG algebras, we would be close to proving the conjecture in full generality. Needless to say, at this point we have no idea how to do that.

The note consists of two parts. In Section 1, we review the relevant parts of Beilinson's conjectures, with an elegant reformulation due to K. Kato presented in Section 2, In Section 3, we state the non-commutative version and show why it is true in the finite-dimensional algebra case.

Acknowledgements. This paper was presented as a talk at the annual MiamiCinvestav-Campinas Homological Mirror Symmetry meeting, and written up during my stay at MSRI during the Non-commutative Geometry program in 2013. I 
am very grateful to the organizers of both events. I am also grateful to the referee for useful suggestions and corrections.

\section{Beilinson conjecture.}

$1.1 \mathbb{R}$-Hodge structures. Recall that an $\mathbb{R}$-mixed Hodge structure $V$ is a triple $\left\langle V_{\mathbb{R}}, V_{\mathbb{C}}, \varphi\right\rangle$ of an $\mathbb{R}$-vector space $V_{\mathbb{R}}$ equipped with an increasing "weight" filtration $W$. a $\mathbb{C}$-vector space $V_{\mathbb{C}}$ equipped with a decreasing "Hodge" filtration $F^{\cdot}$, and an isomorphism $\varphi: V_{\mathbb{R}} \otimes_{\mathbb{R}} \mathbb{C} \cong V_{\mathbb{C}}$ such that $W$. and $F^{\cdot}$ satisfy certain non-degenracy conditions. The category $\mathbb{R}$-MHS of $\mathbb{R}$-mixed Hodge structures is abelian and has homological dimension 1.

An $\mathbb{R}$-mixed Hodge complex is a triple $\left\langle V_{\mathbb{R}}^{\cdot}, V_{\mathbb{C}}^{\cdot}, \varphi\right\rangle$ of a filtered complex $\left\langle V_{\mathbb{R}}^{\cdot}, W_{.}\right\rangle$ of $\mathbb{R}$-vector spaces, a bifiltered complex $\left\langle V_{\mathbb{C}}^{\cdot}, W_{.}, F^{\bullet}\right\rangle$ of $\mathbb{C}$-vector spaces, and a filtered quasiisomorphism

$$
\varphi:\left\langle V_{\mathbb{R}}^{\bullet}, W .\right\rangle \otimes_{\mathbb{R}} \mathbb{C} \cong\left\langle V_{\mathbb{C}}^{\bullet}, W .\right\rangle
$$

such that in every degree $i$, the homology triple $\left\langle H^{i}\left(V_{\mathbb{R}}^{*}\right), H^{i}\left(V_{\mathbb{C}}^{*}\right), \varphi\right\rangle$ with the induced filtrations is an $\mathbb{R}$-mixed Hodge structure.

A map between mixed Hodge complexes is a quasiisomorphism if it induces a quasiisomorphism on the associated graded quotients with respect to filtration. One shows that inverting quasiisomorphisms in the category of $\mathbb{R}$-mixed Hodge complexes, one obtains a triangulated category equivalent to the derived category $\mathcal{D}(\mathbb{R}-\mathrm{MHS})$ (this is closely related to the fact that $\mathbb{R}-\mathrm{MHS}$ has homological dimension 1$)$. For any $\mathbb{R}$-mixed Hodge complex $\left\langle V_{\mathbb{R}}^{\bullet}, V_{\mathbb{C}}^{\bullet}, \varphi\right\rangle$, $\operatorname{Hom}^{\bullet}\left(\mathbb{R}(0), V^{\bullet}\right)$ in $\mathcal{D}(\mathbb{R}-\mathrm{MHS})$ can be computed by the total complex of a bicomplex

$$
W_{0} V_{\mathbb{R}}^{\cdot} \oplus\left(F^{0} V_{\mathbb{C}} \cap W_{0} V_{\mathbb{C}}\right) \longrightarrow W_{0} V_{\mathbb{C}}
$$

where the horizontal differential is $\varphi$ on $W_{0} V_{\mathbb{R}}$ and the embedding map on the second summand.

The category $\mathbb{R}$-MHS is a symmetric tensor category. The Hodge-Tate $\mathbb{R}$ Hodge structure $\mathbb{R}(1)$ of weight 1 is an invertible object in this category, and for any $V^{\bullet} \in \mathcal{D}(\mathbb{R}$-MHS $)$ and any integer $i$, one denotes $V^{\bullet}(i)=V^{\bullet} \otimes \mathbb{R}(1)^{\otimes i}$.

1.2 Absolute Hodge cohomology. Assume given a smooth projective algebraic variety $X$ over $\mathbb{Q}$. Let $X_{a n}$ be the complex manifold underlying the algebraic variety $X \otimes_{\mathbb{Q}} \mathbb{C}$. We then have comparison isomorphisms

$$
H_{D R}^{\cdot}\left(X_{a n}\right) \cong H^{\bullet}\left(X \otimes_{\mathbb{Q}} \mathbb{C}\right) \cong H^{\bullet}(X) \otimes_{\mathbb{Q}} \mathbb{C}
$$


between de Rham cohomology groups. Moreover, by definition, all these groups are equipped with a Hodge filtration, and the comparison isomorphism

$$
\varphi: H^{\bullet}\left(X_{a n}, \mathbb{R}\right) \otimes_{\mathbb{R}} \mathbb{C} \cong H^{\bullet}\left(X_{a n}, \mathbb{C}\right) \cong H_{D R}^{\cdot}\left(X_{a n}\right)
$$

equips the Betti cohomology groups $H^{\bullet}\left(X_{a n}, \mathbb{R}\right)$ with a mixed $\mathbb{R}$-Hodge structure (pure of weight $i$ in degree $i$ ).

One can also refine the comparison isomorphism to a quasiisomorphism

$$
\varphi: C^{\cdot}\left(X_{a n}, \mathbb{R}\right) \otimes_{\mathbb{R}} \mathbb{C} \cong C_{D R}^{\cdot}\left(X_{a n}\right)
$$

between the Betti and the de Rham cohomology complexes, and define an $\mathbb{R}$-mixed Hodge complex

$$
C^{\bullet}(X)=\left\langle C^{\bullet}\left(X_{a n}, \mathbb{R}\right), C_{D R}^{\bullet}\left(X_{a n}\right), \varphi\right\rangle,
$$

where the weight filtration $W$. is the canonical filtration, and the Hodge filtration $F^{\bullet}$ on the de Rham cohomology complex

$$
C_{D R}^{\bullet}\left(X_{a n}\right)=C^{\bullet}\left(X_{a n}, \Omega_{X}^{\bullet}\right)
$$

is induced by the stupid filtration on the de Rham complex $\Omega_{X}{ }_{X}$. The details of how one defines $\varphi$ and the cohomology complexes are completely irrelevant, since the resulting mixed Hodge complex is anyway quasiisomorphic to the sum of its homology objects.

Definition 1.1. For any integer $j$, the absolute Hodge cohomology groups $H_{A H}^{\bullet}(X, \mathbb{R}(j))$ are given by

$$
H_{A H}^{\cdot}(X, \mathbb{R}(j))=\operatorname{Hom}_{\mathcal{D}(\mathbb{R}-\mathrm{MHS})}\left(\mathbb{R}(0), C^{\bullet}\left(X_{a n}\right)(j)\right),
$$

where $C^{\bullet}\left(X_{a n}\right)$ is the $\mathbb{R}$-mixed Hodge complex of (1.2).

Note that by definition, absolute Hodge cohomology groups can be computed by the bicomplex (1.1). We also note that since the Hodge filtration on $C_{D R}^{\cdot}\left(X_{a n}\right)$ starts with $F^{0}$, we have

$$
H_{A H}^{i}(X, \mathbb{R}(j))=0
$$

for $i>2 j$. 
1.3 The conjecture. Beilinson formulates his conjectures in terms of the associated graded quotients of $K$-groups with respect to the $\gamma$-filtration ("motivic cohomology" in current parlance). For our purposes, it will be convenient to stick to $K$-groups themselves.

To formulate the conjecture, one needs one additional ingredient. Consider the category $\mathbb{R}-\mathrm{MHS}^{\iota}$ of mixed $\mathbb{R}$-Hodge structures $\left\langle V_{\mathbb{R}}, V_{\mathbb{C}}, \varphi\right\rangle$ that are equipped with a additional involution $\iota: V_{\mathbb{R}} \rightarrow V_{\mathbb{R}}$ and an anticomplex involution $\iota: V_{\mathbb{C}} \rightarrow$ $V_{\mathbb{C}}$ preserving $F^{\cdot}$ and $W$. and compatible with $\varphi$. This is again a symmetric tensor category of homological dimension one. Equip the Tate object $\mathbb{R}(1)$ with an involution $\iota$ acting by -1 , and let $\mathbb{R}(i)=\mathbb{R}(1)^{\otimes i}, i \in \mathbb{Z}$. The derived category $\mathcal{D}\left(\mathbb{R}-\mathrm{MHS}^{\iota}\right)$ can then again be obtained from an obvious $\iota$-version of the category of mixed Hodge complexes, and for any two such complexes $V_{1}^{\bullet}, V_{2}^{\bullet}$, we have

$$
\mathrm{RHom}_{\mathcal{D}\left(\mathbb{R}-\mathrm{MHS}^{\iota}\right)}^{\cdot}\left(V_{1}^{\bullet}, V_{2}^{\bullet}\right) \cong \operatorname{RHom}_{\mathcal{D}(\mathbb{R}-\mathrm{MHS})}^{\cdot}\left(V_{1}^{\bullet}, V_{2}^{\bullet}\right)^{\iota},
$$

where in the right-hand side, we take invariants with respect to the involution $\iota$ induced by the involutions on $V_{1}^{*}$ and $V_{2}^{*}$.

Assume given a smooth projective algebraic variety $X$ over $\mathbb{Q}$. Note that since $X$ is defined over $\mathbb{Q} \subset \mathbb{R}$, the complexification $X \otimes_{\mathbb{Q}} \mathbb{C}$ carries a natural anticomplex involution $\iota$, and it induces an anticomplex involution of the underlying analytic variety $X_{a n}$. Therefore the mixed Hodge complex $C^{\bullet}\left(X_{a n}\right)$ of Definition 1.1 becomes an object in $\mathcal{D}\left(\mathbb{R}-\mathrm{MHS}^{\iota}\right)$, and the absolute Hodge cohomology groups acquire a natural involution $\iota$. We note that under our convention, $H^{2 i}\left(\mathbb{P}^{n}\right) \cong \mathbb{R}(-i)$ as objects in $\mathbb{R}-\mathrm{MHS}^{\iota}$ for $0 \leq i \leq n$.

Then Beilinson shows that there exists a canonical functorial regulator map

$$
r: K_{i}(X) \otimes_{\mathbb{Z}} \mathbb{R} \rightarrow \bigoplus_{j} H_{A H}^{2 j-i}(X, \mathbb{R}(j))^{\iota}
$$

for any integer $i \geq 0$, and he conjectures that it is an isomorphism for $i \geq 2$.

By virtue of (1.3), the conjecture can be extended to negative $i$, and in this case it is trivially true. The situation is different for $i=0,1$, "critical values" in Beilinson's terminology. This can be easily seen in the following two examples.

- Let $X=\operatorname{Spec} F$ be the spectrum of a number field. Then by Borel Theorem, the ranks of higher $K$-groups of $X$ are given by

$$
\operatorname{dim} K_{i}(X) \otimes_{\mathbb{Z}} \mathbb{R}= \begin{cases}0, & i=2 j, j \geq 1 \\ r_{2}, & i=4 j-1, j \geq 1 \\ r_{1}+r_{2}, & i=4 j+1, j \geq 1\end{cases}
$$


where $r_{1}$ and $r_{2}$ are the number of real and complex embeddings of the field $K$. This matches exactly the right-hand side of (1.4). However, $K_{0}(X)=\mathbb{Z}$, and $K_{1}(F)=F^{*}$, so that $K_{1}(X) \otimes_{\mathbb{Z}} \mathbb{R}$ is infinite-dimensional.

- Let $X$ be an elliptic curve. Then $\operatorname{dim} K_{0}(E) \otimes_{\mathbb{Z}} \mathbb{R}$ is an extremely delicate invariant predicted by the Birch-Swinnerton-Dyer Conjecture; it is expected to be related to the pole order of the $L$-function of $X$ at 1 , and it depends very essentially on the arithmetic properties of $X$. On the other hand, the right-hand side of (1.4) is the same for all elliptic curves.

To solve the obvious problem with $K_{1}(F)$, Beilinson introduces a further conjecture - he conjectures that every smooth projective variety $X$ over $\mathbb{Q}$ has a regular model $X_{\mathbb{Z}}$ projective and flat over $\mathbb{Z}$. He then replaces $K .(X)$ in (1.4) with the image of the natural map $K_{\bullet}\left(X_{\mathbb{Z}}\right) \rightarrow K .(X)$. In the case $X=\operatorname{Spec} F, X_{\mathbb{Z}}$ can be taken to be the spectrum of the ring of integers $\mathcal{O}_{F} \subset F$, and after tensoring with $\mathbb{R}$, the procedure does not affect higher $K$-groups. However, it changes $K_{1}$ drastically: the Dirichlet Unit Theorem says that

$$
K_{1}\left(X_{\mathbb{Z}}\right) \otimes_{\mathbb{Z}} \mathbb{R}=\mathcal{O}_{F}^{*} \otimes_{\mathbb{Z}} \mathbb{R}
$$

has dimension $r_{1}+r_{2}-1$. This is still different from the right-hand side of (1.4), but the difference of dimensions is now only 1 ; incidentally, it coincides with $\operatorname{dim}_{\mathbb{R}} K_{0}\left(X_{\mathbb{Z}}\right) \otimes_{Z} \mathbb{R}$.

To take account of the remaining discrepancies, and to make things plausible for elliptic curves, too, Beilinson includes the so-called "height pairing" into the picture. We will now recall this. However, we prefer to repackage the whole story in a way that the author learned from K. Kato's brilliant lectures at UChicago in 2010 .

\section{Kato's reformulation.}

We again start with linear algebra. Let us denote by $\mathbb{R}$-HS the category of triples $\left\langle V_{\mathbb{R}}, V_{\mathbb{C}}, \varphi\right\rangle$ of an $\mathbb{R}$-vector space $V_{\mathbb{R}}$, a $\mathbb{C}$-vector space $V_{\mathbb{C}}$ equipped witha decreasing filtration $F^{\cdot}$, and an isomorphism $\varphi: V_{\mathbb{R}} \otimes_{\mathbb{R}} \mathbb{C} \cong V_{\mathbb{C}}$. In other words, objects in $\mathbb{R}$-HS are "R-Hodge structures without the weight filtration" - and without any conditions at all on the filtration $F^{*}$. The category $\mathbb{R}-\mathrm{HS}$ is not an abelian category; however, it has a natual structure of an exact tensor category, so that one can define the tensor derived category $\mathcal{D}(\mathbb{R}-\mathrm{HS})$. As in the mixed Hodge structure case, we can also define an " $\mathbb{R}$-Hodge complex" as a triple $\left\langle V_{\mathbb{R}}^{\cdot}, V_{\mathbb{C}}^{\cdot}, \varphi\right\rangle$ of a complex $V_{\mathbb{R}}^{\cdot}$ of $\mathbb{R}$-vector spaces, a complex $V_{\mathbb{C}}$ of filtered $\mathbb{C}$-vector spaces, and a quasiisomorphism $\varphi: V_{\mathbb{R}}^{\bullet} \otimes_{\mathbb{R}} \mathbb{C} \cong V_{\mathbb{C}}^{\cdot}$; then inverting filtered quasiisomorphisms 
in the category of $\mathbb{R}$-Hodge complexes, we obtain the derived category $\mathcal{D}(\mathbb{R}-\mathrm{HS})$. We also have the obvious $\iota$-versions $\mathbb{R}-\mathrm{HS}^{\iota}, \mathcal{D}\left(\mathbb{R}-\mathrm{HS}^{\iota}\right)$ of these categories, and we have the forgetful functors

$$
\begin{array}{ll}
\mathbb{R}-\mathrm{MHS} \rightarrow \mathbb{R}-\mathrm{HS}, & \mathcal{D}(\mathbb{R}-\mathrm{MHS}) \rightarrow \mathcal{D}(\mathbb{R}-\mathrm{HS}), \\
\mathbb{R}-\mathrm{MHS}^{\iota} \rightarrow \mathbb{R}-\mathrm{HS}^{\iota}, & \mathcal{D}\left(\mathbb{R}-\mathrm{MHS}^{\iota}\right) \rightarrow \mathcal{D}\left(\mathbb{R}-\mathrm{HS}^{\iota}\right)
\end{array}
$$

For any $\mathbb{R}$-Hodge complex $V^{\bullet}, \operatorname{Hom}^{\bullet}\left(\mathbb{R}(0), V^{\bullet}\right)$ in $\mathcal{D}(\mathbb{R}-\mathrm{HS})$ can be computed by the total complex of a bicomplex

$$
V_{\mathbb{R}}^{\bullet} \oplus F^{0} V_{\mathbb{C}} \longrightarrow V_{\mathbb{C}}
$$

a version of (1.1) without $W_{0}$, and to obtain Hom ${ }^{\bullet}$ in $\mathcal{D}(\mathbb{R}-\mathrm{HS} \cdot)$, one has to take $\iota$-invariants in this bicomplex.

For any smooth proper variety $X$ over $\mathbb{Q}, C^{\bullet}\left(X_{a n}\right)$ becomes an object of $\mathcal{D}\left(\mathbb{R}-\mathrm{HS}^{\iota}\right)$ by applying the forgetful functor (2.1). The corresponding version

$$
H_{\mathcal{D}}^{\bullet}(X, \mathbb{R}(j))=\operatorname{Hom}_{\mathcal{D}(\mathbb{R}-\mathrm{HS})}\left(\mathbb{R}(0), C^{\bullet}\left(X_{a n}\right)(j)\right)
$$

of absolute Hodge cohomology groups of Definition 1.1 is known as Deligne cohomology of $X$, and it actually predates the absolute Hodge cohomology. As in the absolute Hodge cohomology case, Deligne cohomology can be computed by the bicomplex (2.2), but in this case, one can actually take the cone of the horizontal differential locally on $X_{a n}$, that is, before taking the cohomology complex $C^{\bullet}\left(X_{a n},-\right)$. Then one gets actual complexes $\mathbb{R}(j)$ of sheaves on $X_{a n}$, and one has

$$
H_{\mathcal{D}}(X, \mathbb{R}(j))=H^{\bullet}\left(X_{a n}, \mathbb{R}(j)\right) .
$$

It is in this form that the Deligne cohomology groups were originally introduced by Deligne.

The forgetful functors (2.1) provide natural maps

$$
H_{A H}^{\bullet}(X,-) \rightarrow H_{\mathcal{D}}^{\bullet}(X,-),
$$

so that the regulator map (1.4) induces a regulator map

$$
r: K_{i}\left(X_{\mathbb{Z}}\right) \otimes_{\mathbb{Z}} \mathbb{R} \rightarrow \bigoplus_{j} H_{\mathcal{D}}^{2 j-i}(X, \mathbb{R}(j))^{\iota},
$$

where $X_{\mathbb{Z}}$ is a good $\mathbb{Z}$-model of $X$ which we tacitly assume given. The drawback of Deligne cohomology is that this map clearly has no chance of being an isomorphism for all $i$-one checks easily that the right-hand side does not vanish for $i<0$. This is one of the reasons Beilinson had to refine Deligne cohomology and introduce 
absolute Hodge cohomology. However, Kato handles this discrepancy in a different way.

Namely, note that the complex $C^{\bullet}\left(X_{a n}\right)$ is equipped with a Poincare pairing

$$
C^{\bullet}\left(X_{a n}\right) \otimes C^{\bullet}\left(X_{a n}\right) \rightarrow \mathbb{R}(d)[2 d],
$$

where $d=\operatorname{dim} X$, and both sides are considered as objects of the tensor category $\mathcal{D}\left(\mathbb{R}-\mathrm{HS}^{\iota}\right)$. If one lets

$$
\widetilde{C}^{\bullet}\left(X_{a n}\right)=\bigoplus_{j} C^{\bullet}\left(X_{a n}\right)(j)[2 j]
$$

then this extends to a pairing

$$
\widetilde{C}^{\bullet}\left(X_{a n}\right)^{\iota} \otimes \widetilde{C}^{\bullet}\left(X_{a n}\right)^{\iota} \rightarrow \mathbb{R}(i)[2 i]
$$

for any particular choice of an integer $i$ in the right-hand side. Let us take $i=1$. Then since $\mathcal{D}\left(\mathbb{R}-H S^{\iota}\right)$ is a tensor category, (2.6) induces a pairing

$$
\widetilde{C}_{\mathcal{D}}^{\bullet}\left(X_{a n}\right) \otimes \widetilde{C}_{\mathcal{D}}^{\bullet}\left(X_{a n}\right) \rightarrow \operatorname{RHom} \cdot(\mathbb{R}(0), \mathbb{R}(1)[2]) \rightarrow \mathbb{R}[1]
$$

where we denote

$$
\widetilde{C}_{\mathcal{D}}^{\bullet}\left(X_{a n}\right)^{\iota}=\mathrm{RHom}_{\mathcal{D}\left(\mathbb{R}-\mathrm{HS}^{\iota}\right)}\left(\mathbb{R}(0), \widetilde{\mathbb{C}}^{\bullet}\left(X_{a n}\right)\right),
$$

considered as a complex of $\mathbb{R}$-vector spaces, and we use the identification

$$
\operatorname{Hom}^{1}(\mathbb{R}(0), \mathbb{R}(1)) \cong \mathbb{R}
$$

easily deduced from (2.2).

We note note that $\widetilde{C}_{\mathcal{D}}^{\cdot}\left(X_{a n}\right)$ coincides, up to a quasiisomorphism, with the target of the regulator map (2.3) . Then (2.6) induces a natural map

$$
r^{*}: \widetilde{C}_{\mathcal{D}}^{\bullet}\left(X_{a n}\right)^{\iota} \rightarrow\left(K_{.}\left(X_{\mathbb{Z}}\right) \otimes_{\mathbb{Z}} \mathbb{R}\right)^{*}[1]
$$

to the complex of $\mathbb{R}$-vector spaces dual to $K\left(X_{\mathbb{Z}}\right) \otimes_{\mathbb{Z}} \mathbb{R}$ shifted by 1 . Using this map, we can now formulate the conjecture.

Conjecture 2.1. For any smooth projective variety $X$ over $\mathbb{Q}$ with a regular model $X_{\mathbb{Z}}$ flat and projective over $\mathbb{Z}$, there exists a natural distinguished triangle

$$
K .\left(X_{\mathbb{Z}}\right) \otimes_{\mathbb{Z}} \mathbb{R} \stackrel{r}{\longrightarrow} \widetilde{C}_{\mathcal{D}}^{\cdot}\left(X_{a n}\right)^{\iota} \stackrel{r^{*}}{\longrightarrow}\left(K .\left(X_{\mathbb{Z}}\right) \otimes_{\mathbb{Z}} \mathbb{R}\right)^{*}[1] \longrightarrow
$$

of complexes of $\mathbb{R}$-vector spaces, where $r$ is the regulator map (2.3), and $r^{*}$ is the dual regulator map (2.7). 
We note that for dimension reasons, the conjectural connecting differential

$$
\delta:\left(K .\left(X_{\mathbb{Z}}\right) \otimes_{\mathbb{Z}} \mathbb{R}\right)^{*} \rightarrow K .\left(X_{\mathbb{Z}}\right) \otimes_{\mathbb{Z}} \mathbb{R}
$$

in the triangle (2.8) can only be non-trivial in degree 0. It is further expected to coincide with the inverse to the non-degenerate height pairing on the kernel of the regulator map on $K_{0}\left(X_{\mathbb{Z}}\right) \otimes_{\mathbb{Z}} \mathbb{R}$. This explain the story for elliptic curves: $K_{0}\left(X_{\mathbb{Z}}\right) \otimes_{\mathbb{Z}} \mathbb{R}$ can be large, but the height pairing on the codimension-one subspace of numerically trivial cycles is non-degenerate, so that $\delta$ kills off this subspace. On the other hand, in the case $X=\operatorname{Spec} F, X_{\mathbb{Z}}=\operatorname{Spec} \mathcal{O}_{F}$, the map $\delta$ is actually trivial. The right-hand side of (2.8) vanishes in homological degrees $\geq 2$, while in degrees 0 and 1 we get two exact sequences

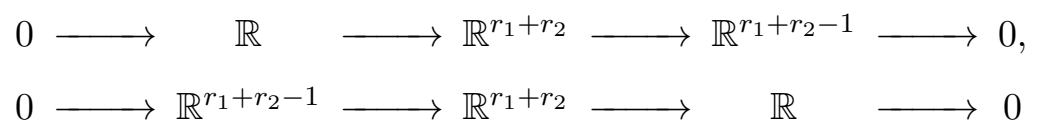

of $\mathbb{R}$-vector spaces, in perfect agreement with Dirichlet Unit Theorem.

\section{Non-commutative version}

3.1 DG algebras. Let us now present a version of Conjecture 2.1 for noncommutative algebraic varieties. To begin with, we should explain what does a "non-commutative algebraic variety" mean; following the current practice, we take it to mean a small DG category considered up to a Morita equivalence, as in $[\mathrm{Ke}$. In fact, in the main conjecture we will even restrict our attention to DG categories with one object, that is, to DG algebras. However, for now, let us recall that for any small DG category $A^{\bullet}$ over a commutative ring $k$, one defines its periodic cyclic homology $H P_{\bullet}\left(A^{\bullet}\right)$. This is the homology of a complex $C P .\left(A^{\bullet}\right)$ of $k$-modules, well-defined up to a quasiisomorphism. We have an additional periodicity quasiisomorphism

$$
u: C P .\left(A^{\bullet}\right) \cong C P .\left(A^{\bullet}\right)[2] .
$$

Moreover, $C P .\left(A^{\bullet}\right)$ can be refined to a filtered complex; the decreasing filtration $F^{\bullet}$ is such that

$$
F^{0} C P .\left(A^{\bullet}\right)=C P^{-}\left(A^{\bullet}\right),
$$

the negative cyclic homology comlpex, and

$$
F^{i} C P .\left(A^{\bullet}\right)=u^{i} F^{0}\left(A^{\bullet}\right)
$$

for any integer $i$. In the filtered sense, (3.1) becomes a filtered quasiisomorphism

$$
u: C P .\left(A^{\bullet}\right) \cong C P .\left(A^{\bullet}\right)(1)[2],
$$


where (1) indicates renumbering of the filtration.

Up to a filtered quasiisomorphism, the complex $C P .\left(A^{*}\right)$ is Morita-invariant - if we have an equivalence $\mathcal{D}\left(A^{\bullet}\right) \cong \mathcal{D}\left(B^{*}\right)$ between the derived category of left modules over two small DG categories $A^{\bullet}, B^{\bullet}$ given by an $A^{\bullet}$ - $B^{\bullet}$-bimodule $M$, then we have a natural identification

$$
C P .\left(A^{\bullet}\right) \cong C P .\left(B^{\bullet}\right),
$$

compatible with the filtration and the periodicity isomorphism (3.2). Moreover, if the base ring $k$ is a field of characteristic 0 , then if the derived category $\mathcal{D}\left(A^{\bullet}\right)$ is equivalent to the derived category $\mathcal{D}(X)$ of quasicoherent sheaves on a smooth proper variety $X$ over $k$, and the equivalence is again given by a bimodule,- loosely speaking, when $A^{*}$ is Morita-equivalent to $X$, - we have a natural identification of filtered periodic complexes

$$
C P .\left(A^{\bullet}\right) \cong \widetilde{C}_{D R}^{\bullet}(X),
$$

where we set

$$
\widetilde{C}_{D R}^{\cdot}(X)=\bigoplus_{j} C_{D R}^{\cdot}(X)(j)[2 j],
$$

as in (2.4). Thus periodic cyclic homology classes can be thought of as a noncommutative generalization of de Rham cohomology classes.

To set up an $\mathbb{R}$-Hodge complex structure on $C P .\left(A^{*}\right)$ in the sense of Section 2 , we also need a version of Betti cohomology for non-commutative varieties, and this is much more problematic. At this point, the best candidate seems to be the semitopological $K$-theory of B. Toën. We will not reproduce its definition here (for a very brief overview with further references, see e.g. [Ka, Section 8]). Let us just say that to any small DG category $A^{\bullet}$ over $\mathbb{C}$, one associates a certain group-like infinite loop space $\mathcal{M}\left(A^{\bullet}\right)$, and one defines the semitopological $K$-groups $K_{\bullet^{s t}}\left(A^{\bullet}\right)$ as

$$
K_{\bullet}^{s t}\left(A^{\bullet}\right)=\pi_{\bullet}\left(\mathcal{M}\left(A^{\bullet}\right)\right) .
$$

Since $\mathcal{M}\left(A^{\bullet}\right)$ is a grouplike infinite loop space, it defines a spectrum, and semitopological $K$-groups are the the homotopy groups of this spectrum. Rationally, every spectrum is an Eilenberg-Maclane spectrum, that is, a complex; therefore we can effectively assume that we are given a complex $K_{\cdot}^{s t}\left(A^{\bullet}\right)_{\mathbb{Q}}=K_{\cdot}^{s t}\left(A^{\bullet}\right) \otimes \mathbb{Q}$ of $\mathbb{Q}$-vector spaces.

Semitopological $K$-theory is compatible with products of DG categories, so that in particular, $K_{\cdot}^{s t}(\mathbb{C})_{\mathbb{Q}}$ is an algebra, and $K_{\cdot}^{s t}\left(A^{*}\right)_{\mathbb{Q}}$ is a module over this algebra. It is easy to prove that in fact,

$$
K^{s t}(\mathbb{C})_{\mathbb{Q}} \cong \mathbb{Q}[\beta],
$$


the algebra of polynomials in one variable $\beta$ of homological degree 2 (see $\mathrm{Ka}$, Corollary 8.4]); the element $\beta$ is a version of the Bott periodicity generator.

For any small DG category $A^{*}$, one can invert the Bott periodicity and consider the complex

$$
K^{s t}\left(A^{\bullet}\right)_{\mathbb{Q}}\left(\beta^{-1}\right)=K_{\cdot}^{s t}\left(A^{\bullet}\right)_{\mathbb{Q}} \otimes_{\mathbb{Q}[\beta]} \mathbb{Q}\left[\beta, \beta^{-1}\right] .
$$

Then a natural functorial Chern character map

$$
K^{s t}\left(A^{\bullet}\right)_{\mathbb{Q}}\left(\beta^{-1}\right) \otimes_{\mathbb{Q}} \mathbb{C} \rightarrow C P .\left(A^{\bullet}\right)
$$

has been constructed by A. Blanc in Bl1. This maps sends $\beta$ to the periodicity endomorphism $u$ on the right-hand side, and it is expected to an isomorphism in good cases. More precisely, there is the following expectation.

Conjecture 3.1 (Blanc-Toën). For any smooth and proper DG category $A^{\bullet}$, the map ch of (3.3) is a quasiisomorphism.

The precise meaning of smoothness and properness for DG categories can be found e.g. in $[\mathrm{Ke}$; let us just say that both properties hold automatically when $A^{\bullet}$ is Morita-equivalent to a smooth projective algebraic variety $X$ over $\mathbb{C}$. In this highly non-trivial special case, the conjecture has been recently proved by Blanc in [Bl2, §4.1]. The general case seems to be wide open.

3.2 The conjecture. To define a non-commutative version of the middle term of the triangle (2.8), we observe that for any DG category $A^{\bullet}$ over $\mathbb{Z}$, the semitopological $K$-theory $K^{s t}\left(A^{\bullet} \otimes \mathbb{C}\right)_{\mathbb{Q}} \otimes_{\mathbb{Q}} \mathbb{C}\left[\beta, \beta^{-1}\right]$ by its very definition carries a natural $\mathbb{R}$-structure (and even a $\mathbb{Q}$-structure). One then has the following two choices:

(i) one can assume Blanc-Toën conjecture, so that the triple

$$
\left\langle K^{s t}\left(A^{\bullet} \otimes \mathbb{C}\right)_{\mathbb{Q}} \otimes_{\mathbb{Q}} \mathbb{R}\left[\beta, \beta^{-1}\right], C P .\left(A^{\bullet} \otimes \mathbb{C}\right), \mathrm{ch}\right\rangle
$$

is an $\mathbb{R}$-Hodge complex in the sense of Section 2, or

(ii) one can define a weak $\mathbb{R}$-Hodge complex by dropping the requirement that the $\operatorname{map} \varphi: V_{\mathbb{R}} \otimes_{\mathbb{R}} \mathbb{C} \rightarrow V_{\mathbb{C}}$ is a quasiisomorphism, and note that the bicomplex (2.2) still makes perfect sense even in this more general situation.

Following the path of least resistance, we will make the second choice. For any small DG category $A^{\bullet}$ over $\mathbb{C}$, we will denote by $C P^{\mathcal{D}}\left(A^{\bullet}\right)$ the total complex of the bicomplex

$$
C P_{\cdot}^{-}\left(A^{\bullet}\right) \oplus\left(K^{s t}\left(A^{\bullet} \otimes \mathbb{C}\right)_{\mathbb{Q}} \otimes_{\mathbb{Q}} \mathbb{R}\left[\beta, \beta^{-1}\right]\right) \stackrel{\text { id }-\mathrm{ch}}{\longrightarrow} C P .\left(A^{\bullet}\right),
$$


where ch is the Chern character map (3.3).

Now, assume given a DG algebra $A^{\bullet}$ over $\mathbb{Z}$, and recall that it defines two small DG categories:

(i) the category $C^{p f}\left(A^{*}\right)$ of perfect modules over $A^{\bullet}$, and

(ii) the category $C_{\bullet^{p s f}}\left(A^{\bullet}\right)$ of pseudoperfect modules over $A^{\bullet}$ - that is, modules that are perfect when considered as modules over $\mathbb{Z}$.

Here $C^{p f}\left(A^{\bullet}\right)$ is Morita-equivalent to $A^{\bullet}$. The category $C^{p s p f}\left(A^{\bullet}\right)$ can be very different, but at least up to a Morita equivalence, it is well-defined. The Hompairing then gives a functor

$$
C_{\bullet}^{p f}\left(A^{\bullet}\right) \times C_{\bullet}^{p s p f}\left(A^{\bullet}\right) \rightarrow C_{\bullet}^{p f}(\mathbb{Z})
$$

into the DG category of perfect complexes of abelian groups, and taking the periodic cyclic homology and semitopological $K$-theory, we obtain a pairing

$$
C P .\left(C_{\cdot}^{p f}\left(A^{\bullet}\right) \otimes \mathbb{C}\right) \otimes C P .\left(C^{p s p f}\left(A^{\bullet}\right) \otimes \mathbb{C}\right) \rightarrow \mathbb{R}(i)[2 i]
$$

compatible with the weak $\mathbb{R}$-Hodge complex structure on both sides. Here $i$ is an arbitrary integer, as in (2.5). Taking $i=1$, we obtain a pairing

$$
C P_{\bullet}^{\mathcal{D}}\left(C_{\bullet}^{p f}\left(A^{\bullet}\right) \otimes \mathbb{C}\right) \otimes C P^{\mathcal{D}}\left(C^{p s p f}\left(A^{\bullet}\right) \otimes \mathbb{C}\right)[1] \rightarrow \mathbb{R},
$$

a version of (2.6).

At the same time, on the $K$-theory side, we have algebraic $K$-groups $K$. $\left(A^{\bullet}\right)$ for any small DG category $A^{\bullet}$ over $\mathbb{Z}$ - for their definition, see e.g. $\mathrm{Ke}$, Subsection 5.2 ] - and we have a Chern character map

$$
K .\left(A^{\bullet}\right) \otimes \mathbb{Q} \rightarrow C P .\left(A^{\bullet} \otimes \mathbb{C}\right) .
$$

This map factor both through $C P_{\cdot}^{-}\left(A^{\bullet} \otimes \mathbb{Q}\right)$ (this is classic) and through $K_{\cdot}^{s t}\left(A^{\bullet} \otimes\right.$ $\mathbb{C})_{\mathbb{Q}} \otimes_{\mathbb{Q}} \mathbb{Q}\left[\beta, \beta^{-1}\right]$ (this has been proved in [Bl1]). Therefore it defines a natural regulator map

$$
r: K_{\bullet}\left(A^{\bullet}\right) \otimes \mathbb{R} \rightarrow C P^{\mathcal{D}}\left(A^{\bullet} \otimes \mathbb{C}\right) .
$$

Moreover, since $A^{\bullet}$ is defined over $\mathbb{Z} \subset \mathbb{R} \subset \mathbb{C}$, the weak $\mathbb{R}$-Hodge complex $C P .\left(A^{\bullet} \otimes \mathbb{C}\right)$ has a natural $\iota$-version, and the regulator map actually gives a map

$$
r: K_{.}\left(A^{\bullet}\right) \otimes \mathbb{R} \rightarrow C P^{\mathcal{D}}\left(A^{\bullet} \otimes \mathbb{C}\right)^{\iota} .
$$


Since $A^{\bullet}$ is Morita-equivalent to $C_{\bullet^{p f}}\left(A^{\bullet}\right)$, we may replace it with $C_{\bullet^{p f}}\left(A^{\bullet}\right)$ in the right-hand side of (3.6). Now define $K^{\prime}\left(A^{\bullet}\right)_{\mathbb{R}}$ by

$$
K^{\prime}\left(A^{\bullet}\right)_{\mathbb{R}}=\left(K_{\bullet}\left(C^{p s p f}\left(A^{\bullet}\right)\right) \otimes \mathbb{R}\right)^{*},
$$

the complex of $\mathbb{R}$-vector spaces dual to $K .\left(C^{\text {pspf }}\left(A^{\bullet}\right)\right) \otimes \mathbb{R}$. Then by virtue of the pairing (3.5), the regulator map $r$ of $(\underline{3.6})$ for the DG category $C^{p s p f}\left(A^{\bullet}\right)$ induces a natural map

$$
r^{*}: C P^{\mathcal{D}}\left(A^{\bullet} \otimes \mathbb{C}\right)^{\iota} \rightarrow K_{\bullet}^{\prime}\left(A^{\bullet}\right)_{\mathbb{R}}[1]
$$

a version of (2.7).

Conjecture 3.2. For any $D G$ algebra $A^{\bullet}$ over $\mathbb{Z}$, we have a natural distinguished triangle

$$
K^{\bullet}\left(A^{\bullet}\right) \otimes \mathbb{R} \stackrel{r}{\longrightarrow} C P^{\mathcal{D}}\left(A^{\bullet} \otimes \mathbb{C}\right)^{\iota} \stackrel{r^{*}}{\longrightarrow} K_{\bullet}^{\prime}\left(A^{\bullet}\right)_{\mathbb{R}}[1] \longrightarrow,
$$

where $r$ and $r^{*}$ are the maps (3.6) and (3.7).

Remark 3.3. For a general DG algebra $A^{\bullet}$, the meaning of the invariant $K^{\prime}\left(A^{\bullet}\right)_{\mathbb{R}}$ in Conjecture 3.2 seems very unclear. If $A^{\bullet}$ is actually smooth and proper over $\mathbb{Z}$, then $C_{\bullet}^{p f}\left(A^{\bullet}\right)$ is Morita-equivalent to $C^{p s p f}\left(A^{\bullet}\right)$, and $K_{-i}^{\prime}\left(A^{\bullet}\right)_{\mathbb{R}}$ are the dual $\mathbb{R}$-vector spaces to $K_{i}\left(A^{*}\right) \otimes \mathbb{R}$. However, in practice, what one cares about are DG algebras smooth and proper over $\mathbb{Q}$, and it is certainly too much to expect that every such DG algebra has a smooth and proper model over $\mathbb{Z}$. Note that in the commutative case, Beilinson only asks for a model $X_{\mathbb{Z}}$ which is regular as an abstract scheme, not smooth over $\mathbb{Z}$. It might very well be that as stated, Conjecture 3.2 admits easy counterexamples, and one needs to impose some version of regularity; unfortunately, at this moment we have no idea what it could possibly be.

Remark 3.4. Every map $f: A^{\bullet} \rightarrow B^{\bullet}$ between DG algebras induces a pair of adjoint functors between the DG categories of DG modules over them. However, only one functor of the pair always sends perfect modules to perfect modules, and the other one always sends pseudoperfect modules to pseudoperfect modules. Therefore we have natural DG functors

$$
f^{*}: C_{\bullet}^{p f}\left(A^{\bullet}\right) \rightarrow C_{\bullet}^{p f}\left(B^{\bullet}\right), \quad f^{*}: C_{\bullet}^{p s p f}\left(B^{\bullet}\right) \rightarrow C_{\bullet}^{p s p f}\left(A^{\bullet}\right) .
$$

Since $K$-groups are functorial with respect to DG functors, this shows that both $K .(-)$ and $K_{.}^{\prime}(-)_{\mathbb{R}}$ are covariantly functorial with respect to DG algebra maps. So are the regulator maps $r$ and $r^{*}$. By adjunction, the Hom-pairing is compatible with functoriality, so that the triangles (3.8) of Conjecture 2.1 should also be covariantly functorial in $A^{\circ}$. 
3.3 Finite-dimensional algebras. Let us now illustrate Conjecture 3.2 in the simple paritucular case of finite-dimensional algebras. We thus assume given a ring $A$ of finite rank as a $\mathbb{Z}$-module, and consider the additive invariants of $A$ considered as a DG algebra over $\mathbb{Z}$.

We first observe that Conjecture 3.1 is almost trivially true for $A^{\bullet}=A \otimes \mathbb{C}$. Here is a sketch of a possible proof.

Sketch of a possible proof of Conjecture 3.1 for $A^{\bullet}=A \otimes \mathbb{C}$. First of all, by the same argument as in [Ka, Lemma 8.3], the infinite loop space $\mathcal{M}(A \otimes \mathbb{C})$ is the group completion of the space

$$
\coprod B \operatorname{Aut}(P)
$$

where the sum is over the isomorphism classes of finitely generated projective $A \otimes \mathbb{C}$-modules $P, \operatorname{Aut}(P)$ stands for the group of automorphisms of such a module $P$ considered as a complex Lie group, and $B \operatorname{Aut}(P)$ is its classifying space. Next, note that since $A \otimes \mathbb{C}$ is a finite-dimensional algebra over $\mathbb{C}$, it is a nilpotent extension of a semisimple algebra $A^{s s}$. Then $A^{s s}$ is the sum of several matrix algebras over $\mathbb{C}$, and since both $K_{\cdot}^{s t}$ and $H P$. are Morita-invariant and compatible with sums, the statement for $A^{s s}$ follows from the corresponding statement for $\mathbb{C}$ itself (that is, $\mathrm{Ka}$, Corollary 8.4]). It remains to notice that on one hand, $H P$. does not change under nilpotent extensions of algebras over a field of characteristic 0 by a theorem of Goodwillie [G], and on the other hand, sending $P$ to $P^{s s}=P \otimes_{A \otimes \mathbb{C}}$ $A^{s s}$ gives a one-to-one correspondence between isomorphism classes of finitely generated projective modules over $A \otimes \mathbb{C}$ and $A^{s s}$, and for any such projective $P$, $\operatorname{Aut}(P)$ is a unipotent extension of $\operatorname{Aut}\left(P^{s s}\right)$, so that the natural map $B \operatorname{Aut}(P) \rightarrow$ $B \operatorname{Aut}\left(P^{s s}\right)$ is a homotopy equivalence.

We now consider Conjecture 3.2, and we note that it can be handled by the same argument. Namely, changing the $\mathbb{Z}$-lattice $A$ in $A \otimes \mathbb{Q}$ does not affect any of the terms in (3.8), and doing such a change if necessary, we may assume that $A$ is a nilpotent extension of an algebra $A^{s s}$ of the form

$$
A^{s s}=\prod_{i} A_{i}
$$

where $A_{i}$ is a maximal order in an algebra $A_{i} \otimes \mathbb{Q}$ over $\mathbb{Q}$, a matrix algebra over a skew-field of finite rank over $\mathbb{Q}$. For $A^{s s}, K_{.}\left(A^{s s}\right) \otimes \mathbb{R}$ and $K^{\prime}\left(A^{s s}\right) \otimes \mathbb{R}$ are dual vector spaces, and they can be computed by the Borel Theorem. Thus verifying the conjecture for $A^{s s}$ is straightforward, and we only need to show that Conjecture 3.2 is stable under nilpotent extensions. For this, one combines the following two observations. 
(i) After tensoring with $\mathbb{Q}$, the cone of the natural map $K .(A) \rightarrow K_{\bullet}\left(A^{s s}\right)$ is identified by the Chern character map with the cone of the natural map $C P_{.}^{-}(A \otimes \mathbb{Q}) \rightarrow C P_{.}^{-}\left(A^{s s}\right)-$ this is a version of the famous Goodwillie Theorem of $[\mathrm{G}]$.

(ii) The other terms in the bicomplex (3.4) do not change inder nilpotent extensions, and by Quillen Devissage Theorem, neither does the right-hand side $K^{\prime}(A \otimes \mathbb{C})_{\mathbb{R}}$ of the triangle $(\underline{3.8})$.

\title{
References
}

[Be] A. Beilinson, Higher regulators and values of L-functions, VINITI Current Problems in Mathematics 24 (1984), Moscow (in Russian).

[Bl1] A. Blanc, Topological K-theory and its Chern character for non-commutative spaces, arXiv:1211.7360

[Bl2] A. Blanc, Invariants topologiques des Espaces non commutatifs, arXiv:1307.6430.

[G] T. Goodwillie, Relative algebraic K-theory and cyclic homology, Ann. of Math. 124 (1986), 347-402.

[Ka] D. Kaledin, Motivic structures in Non-commutative Geometry, Proc. ICM 2010, Vol. II, 461-496; Hindustan Book Agency, New Delhi, 2010.

[Ke] B. Keller, On differential grded categories, Proc. ICM 2006, Vol. II, 151-190; EMS, Zürich, 2006.

\author{
Steklov Math Institute, Algebraic Geometry section \\ AND \\ Laboratory of Algebraic Geometry, NRU HSE
}

E-mail address: kaledin@mi.ras.ru 